\title{
Yams (Dioscorea spp.) for Production of Chips and French Fries ${ }^{1}$
}

\author{
Franklin W. Martin and Ruth Ruberté ${ }^{2}$
}

\section{INTRODUCTION}

Local popularity of potato chips and French fries suggests that suitable substitutes for potato might be found among roots and tubers produced in Puerto Rico for a ready market. Fried plantain chips (platanutre) (2) have been available now for some years, and have become widely known. Sweet potato chips have been developed and sold commercially in several of the states. Instructions for their preparation are given by Boggens and Woodroof (1). Yam, well known and appreciated throughout the Island, should serve equally well for chips and fries. Trials made this year with different species and varieties of yam from the Federal Experiment Station collection indicate that some of them will yield acceptable chips and fries.

\section{MATERIALS AND METHODS}

Twenty-five cultivars of yam from five species of Dioscorea were selected for these tests. They represented varieties well known for their cooking characteristics, but of variable quality. Whole tubers in excellent condition were taken from storage and divided into three sections, young, medium and old portions. The young (lower) and old (upper) portions were peeled by hand and cut into thin slices (about $1.5 \mathrm{~mm}$.) with a simple kitchen device. The two kinds of slices were fried separately in a household deep fat frier until crisp at $375^{\circ} \mathrm{F}$ in commercial corn oil. During frying, the chips were agitated or separated mechanically to assure even cooking. When crisp, the chips were drained, then placed on absorbent paper towels and salted lightly.

After the chips were cooled, some were placed in cellophane bags and stored for 1 week. The remainder were panel-tasted and evaluated on the basis of the following characteristics:

\begin{tabular}{ll}
\hline \multicolumn{1}{c}{ Characteristic } & Measurement \\
\hline Cooking time & Minutes \\
Freedom from tendency to stick & Scale 1-3 \\
Crispness & Scale 1-3 \\
\hline
\end{tabular}

1 Manuscript submitted to Editorial Board October 27, 1971.

2 Plant Geneticist and Research Technician, respectively, Federal Experiment Station, Plant Science Research Division, Agricultural Research Service, USDA, Mayagüez, P.R. 


\begin{tabular}{ll}
\hline \multicolumn{1}{c}{ Characteristic } & Measurement \\
\hline Color acceptability & Scale 1-3 \\
Agreeable flavor & Scale 1-3 \\
Lack of bitterness & Scale 0-3 \\
\hline
\end{tabular}

(A rating of 3 suggests the most desirable degree of the characteristic rated.)

These characteristics were rated again 1 hour later, and again after 1 week.

The center portion of the yam was cut into normal French fries (strips about $0.5-1 \mathrm{~cm}$. wide and thick) and thin French fries (strips about 0.15 $\mathrm{cm}$. thick and $0.3-0.4 \mathrm{~cm}$. wide). These were fried until well-colored, then drained and placed on absorbent paper towels. Thick French fries were evaluated only while hot, as this is considered the normal way of eating them. Thin French fries had many of the characteristics of chips and were rated in the manner for that product.

A quality score was obtained for each variety of yam by adding the individual scores of the five rated characteristics. This score permitted rapid comparison of varieties. In addition they were rated for convenience in the kitchen (size, shape, waste). The ratings were: Not acceptable, poor, acceptable, good, or excellent as sources of fried foods.

As superior varieties became recognized, one of them, Farm Lisbon, was selected for tests with various cooking fats. With other kinds of chips, the type of fat influences flavor, color, and keeping qualities. Cooking temperature for each was reduced from $375^{\circ}$ as necessary to avoid excessive smoking. The chips were cooked to a pleasant color, then rated by an informal panel.

\section{RESULTS}

Varieties of five species were tested (D. alata, D. rotundata, D. esculenta, $D$. bulbifera and $D$. trifida). Their characteristics, are noted in table 1 . Many of these varieties are new to Puerto Rico, but are available from the authors.

All fries of the species $D$. rotundata, D. esculenta, and $D$. bulbifera contained bitter or acrid substances making them unacceptable for chips or fries. One variety of $D$. trifida yielded acceptable chips. The varieties of D. alata produced fries of varied quality. Forastero and Farm Lisbon, as well as a few other varieties, produced excellent chips.

Although some varieties received high quality scores, inadequacy with respect to a single characteristic sometimes downgraded final judgement. For example, the excellent yam Florido yielded rather pale, bland, accept- 
able chips but these lacked the overall quality of some of the other varieties. Many varieties produced chips of an undesirable color, or with blotchy areas representing oxidized portions. Anthocyanin pigmentation discolored the final chips.

Yam varieties that produced good chips also were those that were rated

TABLE 1.-Principal characleristics of chips of yam (Dioscorea spp.) varielies (lower portion) and quality score

\begin{tabular}{|c|c|c|c|c|c|c|c|c|c|c|}
\hline \multirow{3}{*}{ Species } & \multirow{3}{*}{ Variety } & \multirow{3}{*}{$\begin{array}{c}\text { Cooking } \\
\text { time }\end{array}$} & \multicolumn{5}{|c|}{ Quality characteristics! } & \multicolumn{3}{|c|}{ Quality score? } \\
\hline & & & \multirow{2}{*}{ 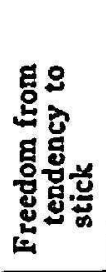 } & \multirow[b]{2}{*}{ 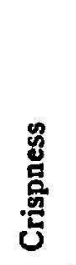 } & \multirow{2}{*}{ 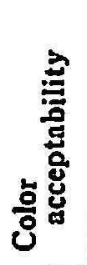 } & \multirow{2}{*}{ 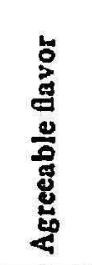 } & \multirow{2}{*}{ 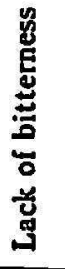 } & \multicolumn{2}{|c|}{ After 1 hour } & \multirow{2}{*}{$\frac{\begin{array}{c}\text { After } 1 \\
\text { week }\end{array}}{\frac{5}{20}}$} \\
\hline & & & & & & & & 递 & 总 & \\
\hline & & Minute & & & & & & & & \\
\hline D. alala & Morado & 8 & 1 & 3 & 3 & 3 & 3 & 13 & 12 & 8 \\
\hline D. alala & Ashmore & 8 & 3 & 3 & 2 & 2 & 3 & 13 & 13 & 11 \\
\hline D. alala & Vino Purple & 5 & 3 & 2 & 1 & 1 & 3 & 10 & 11 & 9 \\
\hline D. alala & Purple Lisbon & 7 & 2 & 3 & 1 & 1 & 3 & 10 & 10 & 8 \\
\hline D. alala & Macorís & 5 & 3 & 2 & 1 & 1.5 & 3 & 10.5 & 11 & 9 \\
\hline D. alala & Florido & 5 & 2 & 3 & 2 & 2 & 3 & 12 & 13 & 11 \\
\hline D. alala & Yellow Lisbon & 7 & 3 & 2 & 2 & 2 & 3 & 12 & 15 & 10 \\
\hline D. alata & $\begin{array}{l}\text { Bottleneck } \\
\text { Lisbon }\end{array}$ & 6 & 3 & 1 & 2 & 3 & 3 & 12 & 15 & 10 \\
\hline D. alata & Oriental & 5 & 3 & 2 & 1 & 1 & 3 & 10 & 10 & 8 \\
\hline D. alaila & Seal Top & 7 & 3 & 3 & 2 & 2 & 3 & 13 & 13 & 12 \\
\hline D. alata & Smooth Statia & 5 & 3 & 2 & 1 & 1 & 3 & 10 & - & 9 \\
\hline D. alata & Farm Lisbon & 7 & 2 & 3 & 3 & 3 & 3 & 14 & 14 & 12 \\
\hline D. alata & Feo & 7 & 3 & 3 & 3 & 3 & 3 & 15 & 15 & 14 \\
\hline D. alata & Gordito & 7 & 3 & 3 & 2 & 2 & 3 & 13 & - & 12 \\
\hline D. alata & $\begin{array}{l}\text { Hawaii } \\
\text { Branched }\end{array}$ & 5 & 3 & 2 & 1 & 2 & 3 & 11 & 11 & 9 \\
\hline D. alala & Forastero & 10 & 3 & 3 & 3 & 3 & 3 & 15 & 15 & 11 \\
\hline D. alala & Hunte & 7 & 3 & 3 & 3 & 3 & 3 & 15 & 11 & 11 \\
\hline D. alala & Vino Blanco & 6 & 3 & 3 & 1 & 1.5 & 3 & 10.5 & 12 & 9 \\
\hline D. rotundala & Guinea Blanco & 7 & 3 & 3 & 3 & 2 & 1 & 12 & 10 & 9 \\
\hline D. rolundala & Guinea Peludo & $\mathbf{5}$ & 3 & 3 & 3 & 1 & 1 & 11 & 9 & 8 \\
\hline D. bulbifera & $\begin{array}{l}\text { Gunda (Sharp } \\
\text { Angled) }\end{array}$ & 7 & 3 & 2 & 2 & 1 & 2 & 10 & - & 8 \\
\hline D. bulbifera & Gunda (round) & 5 & 3 & 1 & 1 & 1 & 0 & 6 & - & 7 \\
\hline D. esculenta & Papa & 6 & 3 & 3 & 3 & 1 & 1 & 11 & 10 & 10 \\
\hline D. esculenta & Pana & 5 & 3 & 2 & 2 & 1 & 0 & 8 & 10 & 7 \\
\hline D. trifida & Mapuey Largo & 6 & 1 & 3 & 3 & 2 & 3 & 12 & 12 & 11 \\
\hline
\end{tabular}

${ }^{1}$ A rating of 3 suggests most desirable degree of characteristic rated.

${ }^{2}$ Sum of quality characteristic ratings; highest scores indicate highest overall quality. 
highly for cooking characteristics. Tubers of these have white, compact flesh with minimum graininess, and have a low tendency for flesh oxidation. The capacity of a yam to yield a good chip thus can be predicted for $D$. alata varieties.

Chip quality sometimes was higher and sometimes lower in the upper portions of the yam (table 1). Quality did not deteriorate in 1 hour. Quality decreased after a week, however, chiefly through loss of crispness in some and flavor changes in all varieties. These changes were not uniform. The best chips were those scaled very carefully in minimum-sized packages. Antoxidants were not applied to the chips (a normal commercial practice).

TABLE 2.-Principal characteristics of French fries of yam (Dioscorea spp.) varieties (center portion) and quality score

\begin{tabular}{|c|c|c|c|c|c|c|c|}
\hline \multirow[b]{2}{*}{ Variety } & \multicolumn{6}{|c|}{ Quality characteristics' } & \multirow[b]{2}{*}{$\begin{array}{l}\text { Quality } \\
\text { score }\end{array}$} \\
\hline & $\begin{array}{c}\text { Cooking } \\
\text { time }\end{array}$ & $\begin{array}{l}\text { Freedom } \\
\text { from } \\
\text { tendency } \\
\text { to stick }\end{array}$ & Crispness & $\begin{array}{c}\text { Color } \\
\text { accepta- } \\
\text { bility }\end{array}$ & $\begin{array}{l}\text { Agreeable } \\
\text { flavor }\end{array}$ & $\begin{array}{c}\text { Lack of } \\
\text { bitterness }\end{array}$ & \\
\hline & Minules & & & & & & \\
\hline Morado & 7 & 2 & 1 & 2 & 2 & $\mathbf{3}$ & 10 \\
\hline Ashmore & 10 & 3 & 3 & 2 & 2 & 3 & 13 \\
\hline Vino Purple & 5 & 3 & 2 & 1 & 1 & 3 & 9 \\
\hline Purple Lisbon & 7 & 3 & 1 & 1 & 1 & 3 & 9 \\
\hline Macorís & 3 & 3 & 1 & 1 & 1.5 & 3 & 9.5 \\
\hline Florido & 6 & 3 & 1 & 2 & 2 & 3 & 11 \\
\hline Yellow Lisbon & 8 & 3 & 1 & 2 & 2 & $\mathbf{3}$ & 11 \\
\hline Bottleneck Lisbon & 7 & 3 & 2 & 3 & 3 & 3 & 14 \\
\hline Oriental & 4 & 3 & 0 & 1 & 1 & 3 & 8 \\
\hline Seal Top & 8 & 3 & 2 & 2 & 2 & 3 & 12 \\
\hline Smooth Statia & 4 & 3 & 1 & 1 & 1 & 3 & 9 \\
\hline Farm Lisbon & 8 & 3 & 1 & 3 & 3 & 3 & 13 \\
\hline Feo & 7 & 3 & 2 & 3 & 3 & 3 & 14 \\
\hline Gordito & 9 & 3 & 2 & 2 & 3 & 3 & 13 \\
\hline Hawaii Branched & 6 & 3 & 1 & 1 & 1 & $\mathbf{3}$ & 9 \\
\hline Forastero & 5 & 3 & 2 & 2 & 3 & 3 & 13 \\
\hline Vino Blanco & 6 & 3 & 1 & 1 & 1 & 3 & 9 \\
\hline Guinea Blanco & 8 & 3 & 3 & 3 & 1 & $\mathbf{0}$ & 10 \\
\hline Guinea Peludo & 6 & 3 & 3 & 3 & 2 & 2 & 13 \\
\hline Gunda (sharp angled) & - & - & - & - & - & - & - \\
\hline Gunda (round) & - & - & - & - & - & - & - \\
\hline Papa & $\mathbf{5}$ & 3 & 2 & 3 & 1 & 1 & 10 \\
\hline Pana & 5 & 3 & 2 & 3 & 1 & 2 & 11 \\
\hline Mapuey Largo & 7 & 3 & 2 & 3 & 2 & 3 & 13 \\
\hline
\end{tabular}

1 A rating of 3 suggests most desirable degree of characteristic rated.

2 Sum of quality characteristic ratings; highest scores indicate highest overall quality. 
Thus some deterioration in quality was expected. The best varieties, however, retained considerable quality even after the 1 week holding period.

Hot French fries were often very good (table 2). The varieties that yielded excellent chips also yielded excellent French fries. Quality of French fries is obviously highly correlated to chip quality. These crisps are a good product, both interesting and appetizing. Their characteristics are not reported in detail here.

Performance of all varieties tested and their final ratings are summarized

TABLE 3.-Advantages and disadvanlages, rating of kitchen convenience, and final judgement of yam (Dioscorea spp.) varieties as French fries and fried chips

\begin{tabular}{|c|c|c|c|c|}
\hline Variety & Advantages & Disadvantages & $\begin{array}{c}\text { Kitchen } \\
\text { conveniencel }\end{array}$ & Rating \\
\hline brado & - & Stickiness & Fair & Acceptable \\
\hline & 一 & Poor & Good & Poor \\
\hline Vino Purple & 一 & Purple color & Fair & Not acceptable \\
\hline Purple Lisbon & - & Purple color & Fair & Not acceptable \\
\hline Macorís & - & $\begin{array}{l}\text { Poor color, } \\
\text { bitter }\end{array}$ & Fair & Not acceptable \\
\hline Florido & - & - & Excellent & Acceptable \\
\hline Yellow Lisbon & Good flavor & Poor shape & Good & Excellent \\
\hline Bottleneck Lisbon & - & Very poor shape & Poor & Excellent \\
\hline Oriental & 一 & Poor flavor & Good & Not acceptable \\
\hline Seal Top & 一 & - & Excellent & Acceptable \\
\hline Smooth Statia & - & Poor color & Excellent & Not acceptable \\
\hline Farm Lisbon & Excellent flavor & Poor shape & Poor & Excellent \\
\hline Feo & $\begin{array}{l}\text { Good color and } \\
\text { flavor }\end{array}$ & Poor shape & Poor & Excellent \\
\hline Gord & - & - & Fair & Acceptable \\
\hline Hawaii Branched & - & Poor color & Fair & Poor \\
\hline Forastero & $\begin{array}{l}\text { Excellent color, } \\
\text { flavor, crisp- } \\
\text { ness }\end{array}$ & - & Good & Excellent \\
\hline Hunt & Good flavor & & Fair & Very good \\
\hline Vino Blanco & - & Poor shape & Fair & Poor \\
\hline Guinea Blanco & $\begin{array}{l}\text { Good color and } \\
\text { crispness }\end{array}$ & Bitterness & Good & Not acceptable \\
\hline Guinea Peludo & $\begin{array}{l}\text { Good color and } \\
\text { crispness }\end{array}$ & Bitterness & Good & Poor \\
\hline $\begin{array}{l}\text { Gunda (sharp } \\
\text { angled) }\end{array}$ & - & Acrid taste & Fair & Not acceptable \\
\hline Gunda (round) & 一 & Acrid taste & & Not acceptable \\
\hline Papa & - & Bitterness & Excellent & Not acceptable \\
\hline Pana & Good color & Bitterness & Excellent & Not acceptable \\
\hline Mapuey Largo & & Stickiness & Fair & Acceptable \\
\hline
\end{tabular}

${ }^{1}$ Kitchen convenience is an estimate closely related to ease of peeling, low waste, and moderate size of tuber. 
in table 3. Some of the best varieties have unaceeptable shapes for agronomic purposes. As a source of fries, the variety Forastero is excellent. This variety combines agronomic vigor with excellent cooking and processing characteristics. Its shape is good, and can probably be adapted to machine harvesting. Forastero is often seen in the Mayagüez markets, especially toward the end of the yam season.

Cooking fat influenced quality of yam chips of the variety Farm Lisbon (table 4). Neither lard nor hydrogenated vegetable oil produced very good chips. Nevertheless, when the two were mixed in proportions of 40:60, respectively, (as recommended by González, ct al. (3) for fried plantain chips), the chips were of excellent flavor. The mixture of lard and hydro-

TABLE 4.-Ratings of four cooking fats in terms of their effects on chips of Dioscorea alata variety Farm Lisbon

\begin{tabular}{|c|c|c|c|c|c|c|c|c|}
\hline \multirow[b]{2}{*}{ Medium } & \multicolumn{8}{|c|}{ Quality characteristics' } \\
\hline & $\begin{array}{l}\text { Cook- } \\
\text { ing } \\
\text { tem- } \\
\text { pera- } \\
\text { ture }\end{array}$ & $\begin{array}{l}\text { Cook- } \\
\text { ing } \\
\text { time }\end{array}$ & $\begin{array}{l}\text { Free- } \\
\text { dom } \\
\text { from } \\
\text { tend- } \\
\text { ency } \\
\text { to stick }\end{array}$ & $\underset{\text { ness }}{\text { Crisp- }}$ & 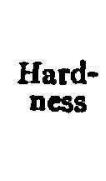 & $\begin{array}{l}\text { Ap- } \\
\text { pear- } \\
\text { ance }\end{array}$ & $\begin{array}{l}\text { Fla- } \\
\text { vor }\end{array}$ & $\begin{array}{c}\text { Net } \\
\text { value }\end{array}$ \\
\hline & $\begin{array}{c}\text { Degrees } \\
\text { F. }\end{array}$ & $\begin{array}{l}\text { Min- } \\
\text { utes }\end{array}$ & & & & & & \\
\hline Corn oil & 375 & 4 & 3 & 1.5 & 2 & 3 & 2.5 & 3 \\
\hline $\begin{array}{l}\text { Hydrogenated vege- } \\
\text { table shortening }\end{array}$ & 325 & 5 & 2 & 2 & 2 & 2 & 1.5 & $\mathbf{2}$ \\
\hline Lard & 300 & 5 & 2 & 2 & 2 & 2 & 2 & 2 \\
\hline $\begin{array}{l}40 \% \text { lard }+60 \% \text { hy- } \\
\text { drogenated vege- } \\
\text { table oil }\end{array}$ & 350 & 5 & 2 & 2 & 2 & 3 & 3 & 2.5 \\
\hline
\end{tabular}

1 A rating of 3 suggests most desirable degree of characteristic rated.

genated vegetable oil was preferred by half the panel members over corn oil as a cooking fat.

\section{DISCUSSION AND CONCLUSIONS}

Although the principal purpose of these tests was to evaluate a number of yam varieties being grown in the Federal Experiment Station collection for their potential use as chips and fries, superior varieties known already in Puerto Rico were found to be excellent sources of chips. Among those is the variety Forastero, for example. Frequent bitterness of Guinea Blanco and a bland product from Florido, do not recommend these otherwise excellent yam varieties for chips.

A high quality yam chip can be prepared for marketing with little additional study. The chief necessity is an antoxidant to avoid flavor changes 
in the fat of the cooked product. We intend to conduct a search for such an antoxidant this coming season. The chip also can be improved by thinner slicing, and adjusting the cooking time as necessary.

As new varicties of yam are added to the federal collection, additional selections will be made for potential use in this manner. Our experience thus far suggests these should be white and dense-fleshed varieties of Dioscorea alata.

\section{SUMMARY}

Tubers of 25 varieties of yam (Dioscorea spp.) were peeled, sliced, and cooked in corn oil at $375^{\circ} \mathrm{F}$.

Chips were evaluated freshly cooked and after 1 week of storage. They were rated for crispness, hardness, flavor, and appearance. Species of yam other than $D$. alala usually did not yield good chips. The Puerto Rican variety Forastero (D. alala) yielded one of the best chips. The quality of hot French fries was related closely to chip quality. Among cooking fats, corn oil and a mixture of lard and hydrogenated vegetable oil gave best results. These results can be commercialized with little modification.

\section{RESUMEN}

Tubérculos de 25 variedades de ñame (Dioscorea spp.) se pelaron, se cortaron en rodajas y se cocieron en aceite de maiz a $375^{\circ} \mathrm{F}$. Las rodajas fritas $\mathrm{u}$ hojuelas se evaluaron inmediatamente después de cocerse y después de 1 semana de almacenamiento. Se observó su dureza, tostadura, sabor y apariencia. De ninguna de las especies, con excepción de $D$. alata, se lograron hojuelas fritas de calidad aceptable. Con la variedad puertorriqueña Forastero $(D$. alata) se obtuvo una de las mejores. La calidad de las papas fritas (estilo francés) calientes estaba estrechamente relacionada con la calidad de las hojuelas fritas. Entre los aceites para cocinar, solamente el aceite de maíz y una mezcla de manteca con aceite vegetal hidrogenado dieron resultados excelentes. Con poca modificación, las técnicas serían útiles para uso industrial.

\section{LITERATURE CITED}

1. Boggens, T. S., Jr., and Woodroof, J. G., Sweet Potato Chips, Ga. Agr. Expt. Sta., Leaflet No. 6 (Rev.), Experiment, Ga., 1964.

2. Cancel, L. E., González, M. A., and Sánchez-Nieva, F., Elaboración del Platanutre, Agr. Exp. Sta. Univ. P.R., Misc. Publ. 6, LTA., 1962.

3. González, M. A., Diaz-Negrón, E., and Sandoval, A. R., Studies on the stability of fried plantain chips, J. Agr. Univ. P.R., 53 (1): 67-74, 1969. 